Check for updates

Cite this: RSC Adv., 2019, 9, 39515

Received 29th September 2019 Accepted 25th November 2019

DOI: 10.1039/c9ra07906h

rsc.li/rsc-advances

\section{A simple one-pot fabrication of silver loaded semi- interpenetrating polymer network (IPN) hydrogels with self-healing and bactericidal abilities $\dagger$}

\begin{abstract}
Mimpin Ginting, (D) *a Indra Masmur, ${ }^{a}$ Subur P. Pasaribu (D) ${ }^{b}$ and Hestina ${ }^{c}$
In the last decade, there has been a significant increase in the development of self-healing hydrogels. However, in most cases, the synthesized self-healing hydrogels possess no antibacterial properties. Further, the preparation of self-healing hydrogels usually requires sophisticated processes and also involves multiple steps. Herein, we proposed a simple one-pot synthesis of silver loaded semi-IPN hydrogels with self-healing and antibacterial properties. The hydrogels were prepared by physical crosslinking between polyacrylic acid (PAA) and ferric ions $\left(\mathrm{Fe}^{3+}\right)$ and further modified by the interpenetration of gelatin-silver in the networks. In addition, the effect by varying the gelatin concentration was also studied. The mechanical properties of the as-prepared hydrogels reached $0.79 \mathrm{MPa}$ in stress and $920 \%$ in strain with the self-healing efficiency of $87.5 \%$ (healed at $70{ }^{\circ} \mathrm{C}$ for $2 \mathrm{~h}$ ). As displayed by the SEM images, the incorporated silver chloride nanoparticles ( $\mathrm{AgCl}$ NPs) in gelatin-free hydrogels were agglomerated. Meanwhile, well-distributed $\mathrm{AgCl}$ NPs in the hydrogels were obtained in the presence of gelatin which acts as a stabilizer. Moreover, due to $\mathrm{Fe}^{3+}$ and $\mathrm{AgCl} \mathrm{NPs,} \mathrm{the} \mathrm{hydrogels} \mathrm{were} \mathrm{able} \mathrm{to} \mathrm{inhibit}$ the growth of bacteria indicated by an inhibition zone $(9-9.6 \mathrm{~mm})$ which was examined toward Escherichia coli via the disk-diffusion method.
\end{abstract}

\section{Introduction}

Hydrogels are constructed of 3D networks of either physically and/or chemically cross-linked polymer chains that can hold a large amount of water in the networks. ${ }^{1,2}$ Lately, hydrogels have been further modified to broaden their applications, such as providing self-healing ability to the hydrogels. The name selfhealing hydrogel indicates that the hydrogel is able to heal or repair itself with or without stimuli after being damaged. The attractive properties of hydrogels (e.g., hydrophilicity, biodegradability, absorptivity, and permeability) including selfhealing ability have led to them being applied in applications of tissue engineering, ${ }^{3}$ drug delivery, ${ }^{4}$ wound healing, ${ }^{5}$ and many other applications. However, the issue of poor mechanical properties leads to a short lifespan of the hydrogels which often hinders and limits their applications.

In the past decade, self-healing hydrogels were mostly fabricated by utilizing non-covalent interactions. For instance,

${ }^{a}$ Department of Chemistry, Faculty of Mathematics and Natural Sciences, Universitas Sumatera Utara, Medan-20155, Indonesia. E-mail: mimpin.ginting@yahoo.com

${ }^{b}$ Department of Chemistry, Faculty of Mathematics and Natural Sciences, Mulawarman University, Samarinda-75123, Indonesia

${ }^{c}$ Department of Chemistry, Universitas Sari Mutiara Indonesia, Medan-20123, Indonesia

$\dagger$ Electronic supplementary information (ESI) available. See DOI: 10.1039/c9ra07906h
Wei, et al. (2013) fabricated autonomous self-healing of polyacrylic acid (PAA) hydrogels induced by the migration of ferric ions $\left(\mathrm{Fe}^{3+}\right) .^{6}$ In 2017 , the dual physically cross-linked double network (DN) hydrogels were synthesized Li, et al. through the hydrogen bonds of cross-linked agar networks and the coordination interactions between $\mathrm{Fe}^{3+}$ and PAA. ${ }^{7}$ Shao, et al. (2017) reported a self-healing hydrogel based on hydrogen bonds between cellulose nanofibrils (CNFs) and PAA, and the dual ionic coordination bonds between $\mathrm{Fe}^{3+}$ and carboxylic groups from PAA and carboxylated CNFs. ${ }^{8}$ Aside from its carboxylic functional groups, PAA was basically chosen as the polymer chains to fabricate self-healing hydrogels because of its excellent mechanical properties. Despite its self-healing ability and mechanical properties, hydrogels are vulnerable to bacteria and as mentioned in the studies above, the antibacterial properties of the prepared hydrogels have not been reported yet. Therefore, designing a hydrogel with good mechanical properties, selfhealing and bactericidal abilities will be an interesting idea which probably would broaden the scope of applications of such hydrogels.

Silver (Ag) has been recognized due to its antimicrobial activity and widely used for antimicrobial materials. The known antimicrobial mechanism is because of the release of silver ions $\left(\mathrm{Ag}^{+}\right)$in which $\mathrm{Ag}^{+}$binds to the bacterial cell wall and penetrates inside to disrupt the metabolic pathways as well as the bacteria DNA replication. ${ }^{\mathbf{9}, 10}$ Silver chloride $(\mathrm{AgCl})$ is one of the silver compounds that release the $\mathrm{Ag}^{+}$slowly due to its low solubility. 
The slow release of $\mathrm{Ag}^{+}$is one of the most important factors for bacteria inhibition, particularly in wound healing application. ${ }^{\mathbf{1 1}}$ Moreover, $\mathrm{AgCl}$ is readily precipitated when there is the presence of chloride ions $\left(\mathrm{Cl}^{-}\right)$and $\mathrm{Ag}^{+}$. Regardless of the ease of $\mathrm{AgCl}$ preparation, the agglomeration of $\mathrm{AgCl}$ particles often occurs upon precipitating. To prevent the agglomeration of $\mathrm{AgCl}$ particles, the stabilizing agents such as chitosan, gelatin, ${ }^{\mathbf{1 2}}$ polyvinylpyrrolidone (PVP), ${ }^{13}$ and etc. are employed. Furthermore, by incorporating such gelatin-stabilized silver into the self-healing hydrogels can answer the issues or challenges mentioned earlier.

Inspiring by the challenges aforementioned, we fabricated the silver loaded semi-interpenetrating polymer networks (IPN) hydrogels with self-healing and antimicrobial properties at the same time via free-radical polymerization as illustrated in Fig. 1. So-called semi-IPN hydrogels, because the first network is created through the ionic interaction of carboxylic groups $(-\mathrm{COOH})$ from PAA and $\mathrm{Fe}^{3+}$, meanwhile, gelatin as the second polymer are only interpenetrated into the first network. ${ }^{\mathbf{1 4}}$ To date, there has not been reported to prepare the silver loaded semi-IPN self-healing hydrogels within a single step. In addition, we also investigated the effect by varying the concentration of gelatin. Finally, the antibacterial activity of the prepared hydrogels was examined toward Escherichia coli.

\section{Materials and methods}

\section{Chemicals}

Acrylic acid $\left(\mathrm{CH}_{2} \mathrm{CHCOOH}, 99.1 \%\right.$, Echo Chemical), iron(III) chloride anhydrous $\left(\mathrm{FeCl}_{3}, 98 \%\right.$, Sigma-Aldrich), gelatin (900070-8, Showa Chemical), silver nitrate $\left(\mathrm{AgNO}_{3}, 99.8 \%\right.$, Aencore Chemical), and ammonium persulfate $\left(\left(\mathrm{NH}_{4}\right)_{2} \mathrm{~S}_{2} \mathrm{O}_{8}, 98 \%\right.$, Sigma-Aldrich) were analytical grade and used as received without further treatment.

\section{Preparation of silver loaded semi-IPN hydrogels}

The silver loaded hydrogels were prepared according to the formula given in Table 1.

The $\mathrm{FeCl}_{3}$ with a known amount was added into a beaker glass containing AA solution and stirred until $\mathrm{FeCl}_{3}$ dissolved. This mixture solution was then heated to $50{ }^{\circ} \mathrm{C}$ followed by adding a various concentration of gelatin with continuous stirring. Afterwards, $\mathrm{AgNO}_{3}$ was added into the mixture solution

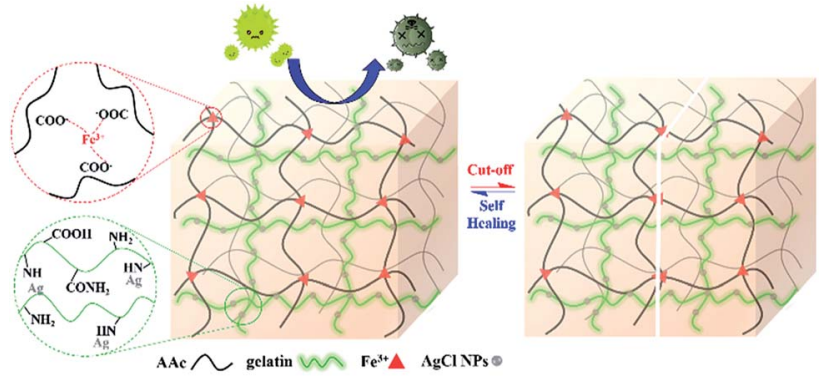

Fig. 1 Schematic illustration of silver loaded semi-IPN hydrogels with self-healing and antibacterial properties.
Table 1 Formulation and nomenclature of silver loaded semi-IPN hydrogels composed of $\mathrm{AA}(30 \mathrm{w} / \mathrm{v} \%), \mathrm{FeCl}_{3}(0.5 \mathrm{~mol} \%$ of $\mathrm{AA}), \mathrm{AgNO}_{3}$ $(0.1 \mathrm{~mol} \%$ of $\mathrm{AA})$, and ammonium persulfate $(0.15 \mathrm{~mol} \%$ of $A A)$ with variation of concentrations of gelatin

Samples Gelatin (wt\% of AA)

\section{Ag-G0}

Ag-G2

Ag-G4

Ag-G6

Ag-G10

Ag-G20

and the obtained mixture solution was stirred for $12 \mathrm{~h}$ at room temperature. Furthermore, nitrogen $\left(\mathrm{N}_{2}\right)$ gas was introduced (degassing) to the as-prepared solution for 30 minutes and immediately added APS into the solution in which the polymerization was conducted for $24 \mathrm{~h}$ at $37^{\circ} \mathrm{C}$. Prior to the characterization and analyses, the obtained gel samples were washed by using DI water.

\section{Chemical functionalities by ATR-FTIR analysis}

AA and gelatin were first made into a thin film by mixing and grinding with $\mathrm{KBr}$ powder (as a reference). Then, the chemical functionalities of the thin film were analyzed by Fourier transform infrared method using a Bio-Rad model FTS-3500GX spectrometer. On the other hand, the surface functional groups of hydrogels were analyzed using such spectrometer equipped with a diamond ATR accessory. All spectra were recorded in the wavenumber of $4000-400 \mathrm{~cm}^{-1}$, a scan number of 128 and a resolution of $8 \mathrm{~cm}^{-1}$.

\section{Degree of swelling (DS) and gel fraction (GF)}

The swelling degree of hydrogels was determined by the gravimetric method in which gel samples were immersed in DI water until reach equilibrium constant weight at $37{ }^{\circ} \mathrm{C}$. Prior to the immersion, gel samples were first weighed as $W_{\mathrm{d}}$ using analytical balance. Afterwards, the water on surface of swollen hydrogels was wiped using blotting tissue. Then, weighed the hydrogels and recorded the weight as $W_{\mathrm{s}}$. The degree of swelling was calculated by eqn (1):

$$
\mathrm{DS}=\frac{W_{\mathrm{s}}-W_{\mathrm{d}}}{W_{\mathrm{d}}} \times 100 \%
$$

Moreover, the determination of gel fraction of hydrogels was conducted by drying such saturated swollen hydrogels until reach equilibrium constant weight at $70{ }^{\circ} \mathrm{C}$. Subsequently, the dried hydrogels were weighed as $W_{\mathrm{d}}^{\prime}$ and the gel fraction was calculated using eqn (2):

$$
\mathrm{GF}=\frac{W_{\mathrm{d}}^{\prime}}{W_{\mathrm{d}}} \times 100 \%
$$


Each determination was run in triplicate and the results were expressed as mean \pm standard deviation (SD).

\section{Mechanical properties and self-healing efficiency}

Firstly, the hydrogels were cut to the size of $6 \mathrm{~cm} \times 1 \mathrm{~cm} \times$ $0.5 \mathrm{~cm}(L \times W \times H)$. The mechanical properties of the asprepared hydrogels were investigated using Testometric M50025AT equipped with a $100 \mathrm{~N}$ load cell and a crosshead speed of $50 \mathrm{~mm} \mathrm{~min}{ }^{-1}$. The results of tensile test on pristine hydrogel were recorded as $\sigma$. Subsequently, the fractured hydrogels were allowed to heal without external stimulation at $70{ }^{\circ} \mathrm{C}$ for $2 \mathrm{~h}$. Afterwards, the healed hydrogels were subjected to tensile test and the results were noted as $\sigma^{\prime}$. Eqn (3) was used to calculate the self-healing efficiency $(\eta)$ of the hydrogels.

$$
\eta=\frac{\sigma}{\sigma^{\prime}} \times 100 \%
$$

The tests were run in triplicate for each specimen and expressed the results as mean \pm standard deviation (SD).

\section{Surface morphology and elemental composition of hydrogels}

Scanning electron microscope (SEM) was performed to observe the surface morphology of hydrogels. Previous to the scanning, hydrogels were freeze-dried for 2 days at a temperature of $-50{ }^{\circ} \mathrm{C}$ and pressure of 10 mTorr. The freeze-dried hydrogels were then adhered on the specimen holder followed by platinum coating through the sputtering process and observed with a JEOL (JSM-6500F, Tokyo, Japan) FE-SEM at an accelerating voltage of $10 \mathrm{kV}$ (magnification of $\times 500$ and $\times 3000$ ). Meanwhile, the energy dispersive X-ray (EDX) spectroscopy was conducted to determine the elemental composition of hydrogel.

\section{XRD analysis}

The crystallinity of the hydrogels was examined using a Bruker D2 Phaser X-ray diffractometer at a scanning rate of $6^{\circ} \mathrm{min}^{-1}$ using $\mathrm{Cu} \mathrm{K} \alpha$ radiation $(\lambda=0.151418 \mathrm{~nm})$ with a working voltage of $30 \mathrm{kV}$ and current of $10 \mathrm{~mA}$. The scanning was collected in the range $2 \theta=10-70^{\circ}$.

\section{Release profile of silver and iron}

To perform the release of silver(I) and iron(III) ions from the hydrogels, each hydrogel samples (Ag-G0-Ag-G20) was placed in a dialysis tubing (Spectra/Por, MWCO $=1000$ Da) and immersed in $20 \mathrm{~mL}$ of phosphate-buffered saline (PBS) solution $(\mathrm{pH}=7.4)$ at $37^{\circ} \mathrm{C}$. After certain time intervals between 0 and $48 \mathrm{~h}$, aliquots $(1 \mathrm{~mL})$ of the sample solution were taken from the release medium and an equivalent amount of fresh buffer was added to maintain a constant volume. The amount of released silver and iron ions in the PBS solution was measured as cumulative release and determined by ICP-AES TY2000.

\section{Antibacterial experiments}

The antibacterial ability of hydrogels was examined toward Escherichia coli (Gram-negative bacteria) using disk diffusion method. Firstly, a single culture of bacteria is added into a glass tube containing $5 \mathrm{~mL}$ Luria-Bertani (LB) broth and inoculated for $12-24 \mathrm{~h}$ at $37^{\circ} \mathrm{C}$ with $200 \mathrm{rpm}$ continuous stirring. Then, the solution containing bacteria was diluted to an $\mathrm{OD}_{600}$ of 0.05 using JASCO V-630 UV/VIS spectrophotometer. In this experiments, positive control of $10 \mu \mathrm{L} \mathrm{H}_{2} \mathrm{O}_{2} 2500$ ppm on filter paper was used and the samples including the filter paper were cut to size of $4 \mathrm{~mm}$ in diameter. Moreover, the samples were adhered on the LB agar plate which has been suspended by E. coli and incubated with face-down position for $24 \mathrm{~h}$ at $37^{\circ} \mathrm{C}$. The average zone of inhibition was observed and measured at 6, 12, 18 and $24 \mathrm{~h}$ of incubation. Similarly, the $5 \mathrm{~mL}$ bacteria-containing LB broth with an $\mathrm{OD}_{600}$ of 0.05 was added into tube with samples. After $24 \mathrm{~h}$ of incubation at $37{ }^{\circ} \mathrm{C}$, the $\mathrm{OD}_{600}$ value of the suspension was measured by UV-Vis spectrophotometer and the experiments were conducted in triplicate.

\section{Results and discussion}

\section{Preparation of silver loaded semi-IPN hydrogels}

In this study, we synthesized the silver loaded semi-IPN hydrogels in one-step preparation and studied the effect by varying gelatin concentration. Firstly, $\mathrm{AgCl}$ is readily precipitated when $\mathrm{AgNO}_{3}$ added into the mixture solution containing $\mathrm{FeCl}_{3}$ followed by the stabilization by gelatin. Subsequently, APS initiator was used to initiate the free-radical polymerization (FRP) of AA to PAA and further formed the 3D polymer networks by physical cross-linking between $\mathrm{COO}^{-}$from PAA with $\mathrm{Fe}^{3+}$. At the same time, the gelatin-stabilized silver penetrated in the network forming semi-IPN during the formation of hydrogel networks. Such hydrogels demonstrated self-healing ability due to the ionic interaction $\left(\mathrm{COO}^{-}-\mathrm{Fe}^{3+}\right)$ as shown in Fig. 2a-c which is also resistant to stretching and twisting force (Fig. 2d and e). In addition, the images observed by the optical microscope proved that the self-healing is occurred indicated by the attached-interfaces of two separated halves. Meanwhile, the particles in the images might be originated from the silver particles.

\section{Chemical functionalities}

The characteristic groups of atoms (functional groups) of silver loaded semi-IPN hydrogels were identified by ATR-FTIR analysis and the result was represented in Fig. 3. The FTIR spectrum of

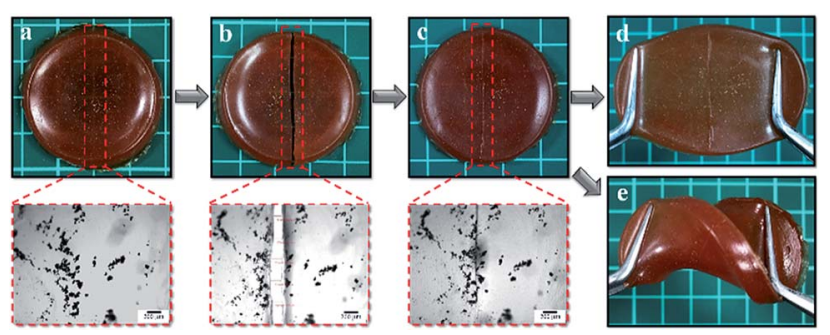

Fig. 2 Photographs of (a) pristine Ag-G0; (b) cut-off Ag-G0; (c) healed Ag-G0; (d) stretched Ag-G0; (e) twisted Ag-G0 and their images of optical microscope (red dashed-line). 


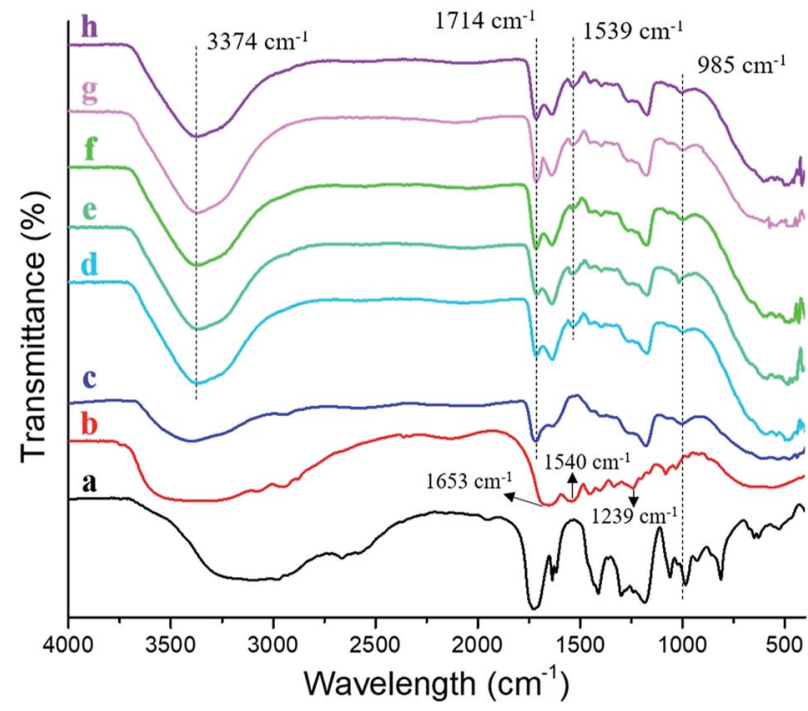

Fig. 3 FTIR spectra of (a) acrylic acid; (b) gelatin; (c) Ag-G0; (d) Ag-G2; (e) Ag-G4; (f) Ag-G6; (g) Ag-G10; and (h) Ag-G20 hydrogels.

AA (Fig. 3a) displayed peaks at $\sim 3100 \mathrm{~cm}^{-1}, 1728 \mathrm{~cm}^{-1}$, and $1633 \mathrm{~cm}^{-1}$ which corresponded to $\nu \mathrm{O}-\mathrm{H}, \nu \mathrm{C}=\mathrm{O}$, and $\nu \mathrm{C}=\mathrm{C}$, respectively. As to gelatin (Fig. 3b), the bands corresponded to stretching vibration of amide I $(\nu \mathrm{C}=\mathrm{O}), \delta \mathrm{N}-\mathrm{H}$ of amide II and amide III were found at $1653 \mathrm{~cm}^{-1}, 1540 \mathrm{~cm}^{-1}$, and $1239 \mathrm{~cm}^{-1}$, respectively, in agreement with previous report. ${ }^{15,16}$ Moreover, the absorption peak at $\sim 3300 \mathrm{~cm}^{-1}$ was assigned to $\nu \mathrm{N}-\mathrm{H}$. Compared to AA, all spectrum of the hydrogels (Fig. 3c-h) indicated a decrease in intensity at $985 \mathrm{~cm}^{-1}$ which is $\delta \mathrm{C}=\mathrm{C}-\mathrm{H}$ and also proved the polymerization of AA. Subsequently, the ionic interaction between carboxylic groups $(-\mathrm{COOH})$ from PAA and $\mathrm{Fe}^{3+}$ was evidenced by the absorption peak at $1714 \mathrm{~cm}^{-1}$. Furthermore, what does distinguish between the gelatin-free (Fig. 3c) and semi-IPN hydrogels (Fig. 3d-h) are the functional groups of amide II at $1539 \mathrm{~cm}^{-1}$ and N-H stretching band at $3374 \mathrm{~cm}^{-1}$ which is overlapped with O-H stretching. Such peaks confirmed the presence of gelatin in the hydrogel (semi-IPN) which was not found in the FTIR spectrum of gelatin-free hydrogel. Meanwhile, there is no alteration for the chemical functionalities by varying the concentration of gelatin.

\section{Degree of swelling and gel fraction}

The results of swelling degree and gel fraction were plotted as a graph and shown in Fig. 4. Firstly, the swelling ratio of Ag-G0 hydrogel was found to be $172.1 \%$ and keep decreasing to $31.9 \%$ with increasing the concentration of gelatin to $20 \mathrm{wt} \%$. The AgG0 hydrogel could swell a considerable amount of water is because the networks of the hydrogel are mainly composed of polyacrylic acid which is hydrophilic owing to its carboxylic groups $(-\mathrm{COOH})$. Furthermore, this functional groups of $-\mathrm{COOH}$ will be deprotonated to anion carboxylic groups $\left(-\mathrm{COO}^{-}\right)$if the $\mathrm{pH} \geq \mathrm{p} K_{\mathrm{a}}(4.2)$, thus led to electrostatic repulsion and loosen the networks in the hydrogel to be filled with more water. ${ }^{17,18}$ However, when gelatin was interpenetrated in the hydrogel networks, the result indicated that gelatin could

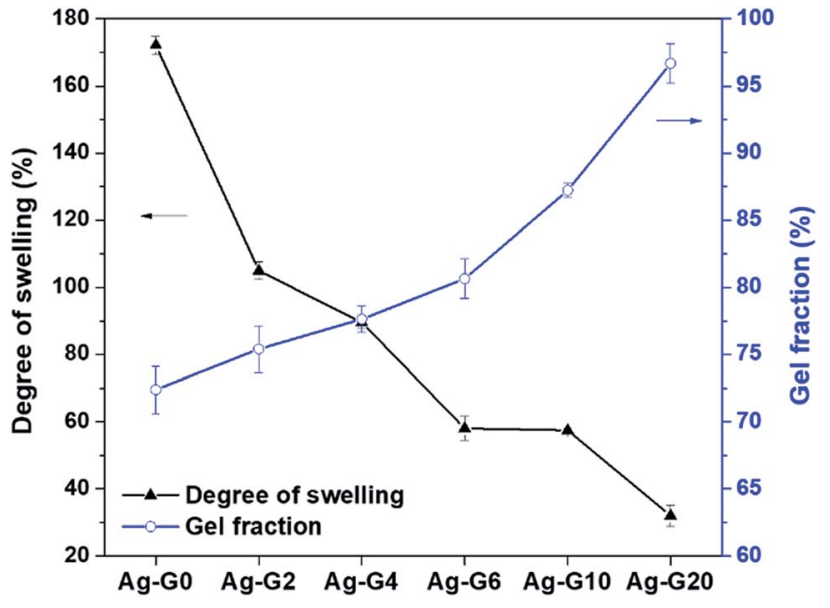

Fig. 4 Degree of swelling and gel fraction of hydrogels with different composition of gelatin.

weaken the water retention capacity of hydrogel. This is probably due to such networks tend to be filled by gelatin rather than water. On the other hand, the gel fraction demonstrated an increasing value $(72-97 \%)$ as the gelatin concentration increases from 0 to $20 \mathrm{wt} \%$. In view of this result, it was probably due to the hydrogel networks and gelatin are interlaced forming the insoluble fraction, therefore increases the gel fraction of hydrogel. The results of both swelling degree and gel fraction of the semi-IPN hydrogels showed a good agreement with the results reported by Hago and Li (2013). ${ }^{19}$

\section{Mechanical properties and self-healing efficiency}

The self-healing efficiency of self-healing hydrogels was quantified by means of uniaxial tensile tests. As shown in Fig. 5a, AgG0 hydrogel exhibited satisfyingly mechanical properties with the tensile stress of $0.79 \mathrm{MPa}$. As to semi-IPN hydrogels, when gelatin was introduced and to further increase from 2 to $10 \mathrm{wt} \%$, a declining values of tensile stress $(\sigma)$ were obtained, which are $0.62,0.51,0.47,0.32$ and $0.25 \mathrm{MPa}$ for Ag-G2, Ag-G4, Ag-G6, AgG10, and Ag-G20, respectively. However, when much more gelatin $(20 \mathrm{wt} \%)$ was used, both tensile stress and strain showed no significant alteration which might indicate that the interpenetration of gelatin into the hydrogel reaches a saturation point. Moreover, as known, gelatin possesses poor mechanical properties, ${ }^{20}$ therefore with increasing gelatin concentration,
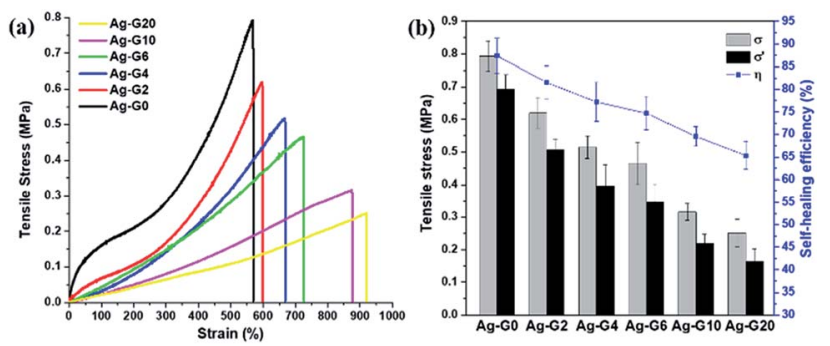

Fig. 5 (a) Tensile stress-strain curves; and (b) the self-healing efficiency of hydrogels with various concentration of gelatin. 
decreases the tensile stress of the obtained hydrogels. Regardless of their tensile stress, the interpenetrated gelatin in hydrogels indicated an improvement in tensile strain. Initially, the tensile strain of Ag-G0 was 570\% and gradually improved to $598,666,725,875$, and $920 \%$ at gelatin concentration of $2,4,6$, 10 , and $20 \mathrm{wt} \%$, respectively. The fractured hydrogels were allowed to heal thereafter at $70{ }^{\circ} \mathrm{C}$ for $2 \mathrm{~h}$ without any external stimuli. Afterwards, the healed hydrogels were subjected to tensile tests again. On average, the healed hydrogels exhibited tensile stress $\left(\sigma^{\prime}\right)$ around $\frac{3}{4}$ of its tensile stress of original hydrogels $(\sigma)$. Finally, the calculated self-healing efficiency of hydrogels $(\eta)$ was $87.4,81.5,77.2,74.7,69.6$, and $65.3 \%$ for AGG0, Ag-G2, Ag-G4, Ag-G6, Ag-G10, and Ag-G20, respectively as shown in Fig. 5b. Apart from weakening the mechanical properties, the increase in gelatin concentration also led to less effective self-healing of the hydrogels. Besides, the cut-off hydrogel should be self-healed along the interfaces due to the ionic interaction between $\mathrm{COO}^{-}$and $\mathrm{Fe}^{3+}$, however, we suspected that such healing is more hindered at higher concentration of gelatin.

\section{SEM and EDX analyses}

Fig. 6a-f depicted the surface morphology of silver loaded hydrogels at the magnification of $\times 500$ and $\times 3000$ including the photographs of the resultant hydrogels (bottom-left-corner). The photographs indicated that the gelatin-free hydrogel, AgG0, possessed orange colour with low transparency while the hydrogels containing gelatin, Ag-G2-Ag-G20, completely showed no transparency. The agglomeration of silver nanoparticles was obtained in Ag-G0 hydrogel as can be seen in Fig. 6a. However, by utilizing gelatin, the silver nanoparticles are distributed uniformly in the hydrogels and seemed to be more uniform with increasing gelatin concentration as can be observed in Fig. 6b-f. In view of this result, gelatin successfully proved its ability as capping agent to stabilize the silver nanoparticles by preventing the agglomeration of silver nanoparticles through its amine functional groups. ${ }^{\mathbf{1 2}}$ Likewise, Russell (1967) reported that the silver is bound to gelatin at low $\mathrm{pH}(\sim 1)$ and in our experiments the measured $\mathrm{pH}$ of solution is $\sim 1.3 .^{21}$

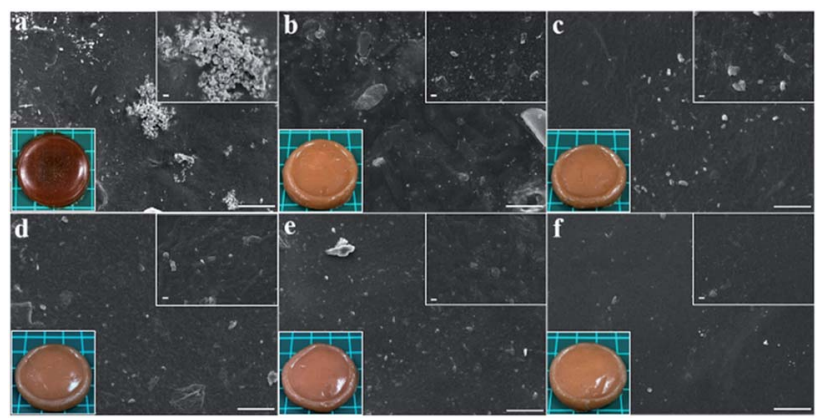

Fig. 6 Surface morphology of (a) Ag-G0; (b) Ag-G2; (c) Ag-G4; (d) AgG6; (e) Ag-G10; and (f) Ag-G20 hydrogels at magnification of 500x (with inserted images at $3000 \times$ and photographs of hydrogels). Scale bars are $50 \mu \mathrm{m}$.
Meanwhile, the result of EDX analysis was represented in Fig. 7 and the quantitative elemental composition was summarized in Table 2. The EDX spectra showed that the elemental composition of the hydrogels composed of $\mathrm{Ag}$ and $\mathrm{Cl}$ elements which also revealed that the silver nanoparticles incorporated in the hydrogels are silver chloride nanoparticles (AgCl NPs).

\section{XRD analysis}

To further confirmed the type of silver incorporated in the hydrogel, XRD analysis was carried out. All of the hydrogels possessed a broad peak at $2 \theta=21^{\circ}$ which is attributed to the polymer networks in the hydrogels (Fig. 8). The six diffraction sharp peaks were obtained at angles $2 \theta=27.8^{\circ}, 32.2^{\circ}, 46.2^{\circ}$, $54.9^{\circ}, 57.6^{\circ}$ and $67.5^{\circ}$ which are ascribed to (111), (200), (220), (311), (222), and (400) planes of the face-centered cubic (fcc) packing of AgCl particles according to JCPDS data (card no. 311238). Correspondingly, the result of XRD analysis also echoed the result of EDX analysis in which the silver nanoparticles incorporated in the hydrogel are AgCl. However, the crystallinity of hydrogels by varying the concentration of gelatin showed similar results.

\section{Release profile of silver and iron}

The release behaviour of silver(I) and iron(III) ion was shown in Fig. 9a and b, respectively. The release profile of silver(I) of semiIPN hydrogel (Ag-G2-Ag-G20) exhibited a similar result in which the burst release occurred at the beginning of immersion and subsequently followed by the sustained release. However, after immersion for $24 \mathrm{~h}$, the result indicated a saturation in releasing the silver(I) ion. Meanwhile, Ag-G0 demonstrated a slightly different release behaviour of silver ion. This is probably due to the agglomerated AgCl NPs. The cumulative release of silver(I) ion was ranging from $75.7-82.5 \%$. On the other hand, the release of iron(III) ion after immersion for $48 \mathrm{~h}$

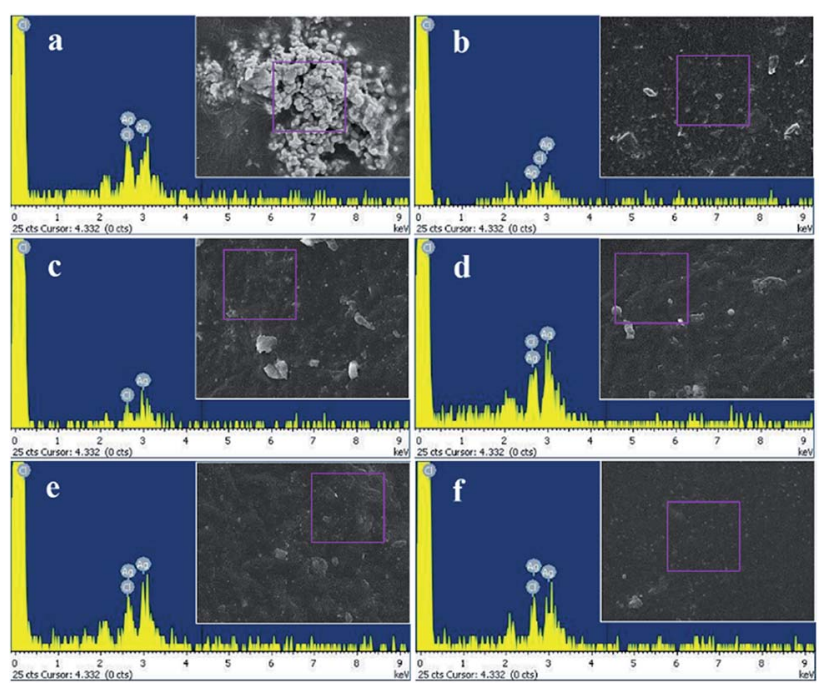

Fig. 7 Elemental composition by EDX analysis of (a) Ag-G0; (b) Ag-G2; (c) Ag-G4; (d) Ag-G6; (e) Ag-G10; and (f) Ag-G20 hydrogels. 
Table 2 The elemental composition of the hydrogels by EDX analysis

\begin{tabular}{llll}
\hline & \multicolumn{2}{l}{ Elements (atomic\%) } & \\
\cline { 2 - 3 } Hydrogels & $\mathrm{Ag}$ & $\mathrm{Cl}$ & Total (\%) \\
\hline Ag-G0 & 67.12 & 32.88 & \multirow{2}{*}{100} \\
Ag-G2 & 50.79 & 49.21 & \\
Ag-G4 & 51.33 & 48.67 & \\
Ag-G6 & 58.62 & 48.67 & \\
Ag-G10 & 54.08 & 45.92 & \\
Ag-G20 & 50.77 & 49.23 &
\end{tabular}

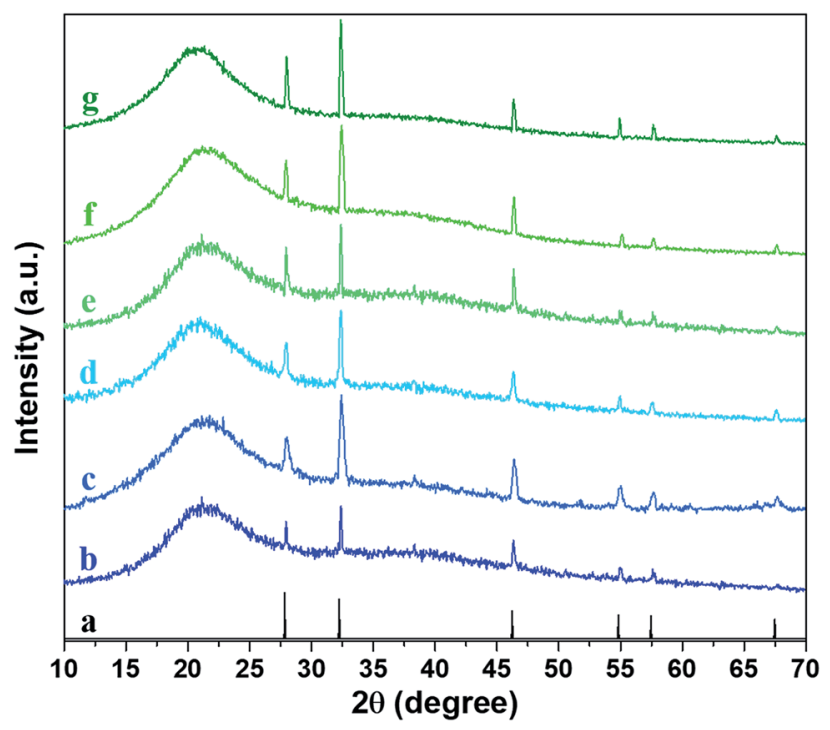

Fig. 8 XRD patterns of (a) AgCl (JCPDS \#31-1238); (b) Ag-G0; (c) AgG2; (d) Ag-G4; (e) Ag-G6; (f) Ag-G10; and (g) Ag-G20 hydrogels.
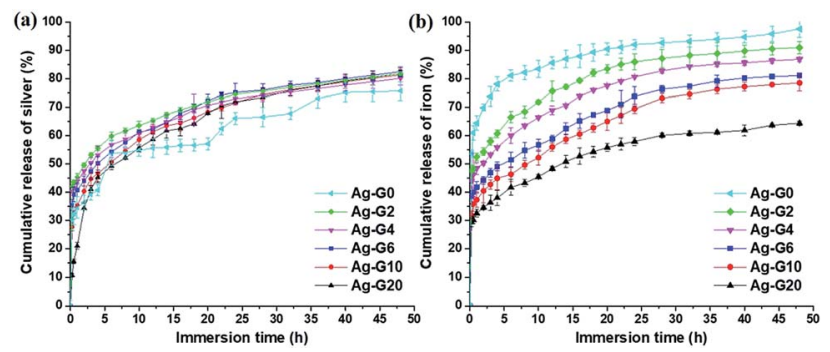

Fig. 9 The cumulative release profile of (a) silver(I) and (b) iron(III) ions from the hydrogel in PBS solution at $\mathrm{pH} 7.4$ as a function of immersion time.

was 97.5, 90.9, 86.8, 81.1, 78.5, and 64.3\% for Ag-G0, Ag-G2, AgG4, Ag-G6, Ag-G10 and Ag-G20, respectively. These results revealed that the cumulative release of iron(III) ion become more sustainable and slower with increasing concentration of gelatin. Consequently, the lowest release profile was demonstrated by the free-gelatin hydrogel. Moreover, the release of iron(III) ion result showed good agreement with the result of gel fraction indicating that the hydrogels with higher gel fraction (less soluble part) release iron(III) ion slower.

\section{Antibacterial activity}

The antibacterial properties of silver-loaded hydrogels with different concentration of gelatin were determined by measuring the average zone of inhibition as a function of incubation time $(t=6,12,18$, and $24 \mathrm{~h})$ toward $E$. coli which has been suspended on LB agar plate. The results of antibacterial tests were depicted in Fig. 10a-e and their average zone of inhibition were given in Table 3. The growth of bacteria on LB agar plate and the inhibition zone of samples could be observed after incubated for $6 \mathrm{~h}$ and become denser and clearer as prolonging the incubation time to 12,18 and $24 \mathrm{~h}$. During the incubation from $6 \mathrm{~h}$ to 12, 18, and $24 \mathrm{~h}$, the zone of inhibition of hydrogels kept decreasing. This is due to the antibacterial activity of the hydrogels is bacteriostatic that prevent the growth of bacteria and is reversible. ${ }^{22}$ Likewise, the result from measurement of $\mathrm{OD}_{600}$ indicated that the hydrogels inhibited the growth of E. coli (Fig. 10f) and the absorbance of bacteriacontaining medium for all tested hydrogels showed no significant difference which is varied between 0.085 and 0.129. Furthermore, the antibacterial activity between the free-gelatin and variation concentrations of gelatin hydrogels showed insignificant difference. The antibacterial activity of the

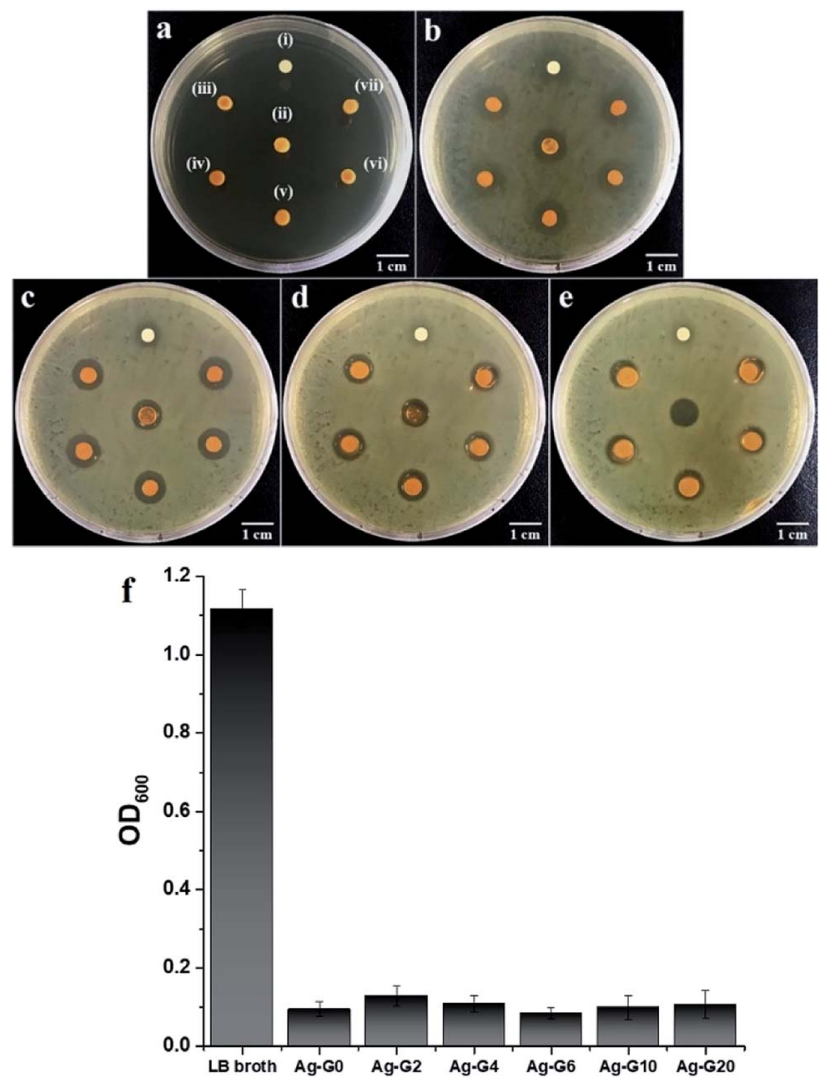

Fig. 10 Antibacterial tests toward Escherichia coli (a) before incubation; incubated for (b) $6 \mathrm{~h}$; (c) $12 \mathrm{~h}$; (d) $18 \mathrm{~h}$; and (e) $24 \mathrm{~h}$ at $37^{\circ} \mathrm{C}$ with the samples of (i) $\mathrm{H}_{2} \mathrm{O}_{2}$; (ii) Ag-G0; (iii) Ag-G2; (iv) Ag-G4; (v) Ag-G6; (vi) $\mathrm{Ag}-\mathrm{G} 10$; and (vii) $\mathrm{Ag}-\mathrm{G} 20$ hydrogels. (f) Absorbance, as measured at $600 \mathrm{~nm}$ as a function of hydrogel samples in bacteria-containing medium after $24 \mathrm{~h}$ of incubation. 
Table 3 The average zone of inhibition of hydrogels toward Escherichia coli

Zone of inhibition (mm)

\begin{tabular}{lllll} 
Samples & $6 \mathrm{~h}$ & $12 \mathrm{~h}$ & $18 \mathrm{~h}$ & $24 \mathrm{~h}$ \\
\hline $\mathrm{H}_{2} \mathrm{O}_{2}$ & 8.95 & 6.33 & 6.27 & 6.13 \\
$\mathrm{Ag}-\mathrm{G} 0$ & 9.78 & 9.09 & 8.64 & 8.10 \\
$\mathrm{Ag}-\mathrm{G} 2$ & 9.90 & 9.49 & 8.98 & 8.09 \\
$\mathrm{Ag}-\mathrm{G} 4$ & 9.99 & 9.67 & 9.34 & 8.59 \\
$\mathrm{Ag-G6}$ & 9.88 & 9.62 & 9.12 & 8.85 \\
$\mathrm{Ag}-\mathrm{G} 10$ & 9.80 & 9.74 & 9.27 & 8.47 \\
Ag-G20 & 9.87 & 9.61 & 9.14 & 8.09
\end{tabular}

hydrogels was originated from the released of silver ions $\left(\mathrm{Ag}^{+}\right)$in the bacterial cultural medium. The $\mathrm{Ag}^{+}$ions would then penetrate into the bacterial through negatively charged cell membrane, caused disruption and led to the death of bacteria. ${ }^{23,24}$ On the other hand, as can be seen in Fig. 1S, $\dagger$ the result of antibacterial experiment with variation $\mathrm{FeCl}_{3}$ concentration revealed that the physical cross-linker of hydrogel, ferric ions $\left(\mathrm{Fe}^{3+}\right)$, also could inhibit the bacterial growth through Fenton oxidation $^{25}$ and the zone of inhibition of the hydrogel increased with increasing concentration of $\mathrm{FeCl}_{3}$. Moreover, the bacteria would adsorb $\mathrm{Fe}^{3+}$ and reduce it to ferrous ions $\left(\mathrm{Fe}^{2+}\right)$ followed by the reaction with $\mathrm{H}_{2} \mathrm{O}_{2}$ to produce reactive species, hydroxyl radical $(\cdot \mathrm{OH})$. This $\cdot \mathrm{OH}$ species caused the breakage of protein and nucleic acids of the bacteria cell. ${ }^{26,27}$ Correspondingly, such phenomenon caused a degradation of the networks of hydrogel and resulted in liquid-like gel Ag-G0 as can be observed in Fig. 10e(ii). Nevertheless, the presence of gelatin could enhance the stability of hydrogel after exposing to the bacteria medium at longer time as can be seen in Fig. 10c-e.

\section{Conclusion}

We have successfully synthesized a silver loaded semi-IPN hydrogel in one-step preparation which is self-healable and exhibit antimicrobial properties. The semi-IPN of gelatin in the PAA-Fe hydrogel network is confirmed by FTIR result in which the functional groups of amide II and $\mathrm{N}-\mathrm{H}$ stretching band from gelatin present at $1539 \mathrm{~cm}^{-1}$ and $3374 \mathrm{~cm}^{-1}$, respectively. Meanwhile, the self-healing ability is facilitated by the ionic interaction between carboxylic groups $(-\mathrm{COOH})$ from PAA and $\mathrm{Fe}^{3+}$ which is proved by the shifting of wavelength from $1728 \mathrm{~cm}^{-1}$ in AA spectrum to $1714 \mathrm{~cm}^{-1}$ in hydrogels spectra. As displayed by SEM images, gelatin proves its ability as a stabilizing agent indicated by the well-distributed silver chloride nanoparticles (AgCl NPs) in the hydrogels. Due to the incorporated $\mathrm{AgCl}$ NPs, the hydrogels inhibit the growth of $E$. coli which is synergistically enhanced by the physical cross-linker, $\mathrm{Fe}^{3+}$, via Fenton oxidation. Overall, the only advantage by adding gelatin in the hydrogel is to stabilize the agglomeration of $\mathrm{AgCl}$ NPs. However, when increasing the amount of gelatin, the mechanical properties of hydrogel decreases and showed no significant difference in antibacterial activity. Hence, the optimum formulation for the semi-IPN hydrogel is Ag-G2 in which such amount of gelatin is enough to stabilize the agglomeration $\mathrm{AgCl}$ NPs in the hydrogel and possesses satisfyingly mechanical properties and self-healing efficiency as well compared to the semi-IPN hydrogel with higher amount of gelatin. Lastly, such hydrogels with self-healing and antimicrobial properties indicate potential applications in biomedical related fields.

\section{Conflicts of interest}

There are no conflicts to declare.

\section{Acknowledgements}

The authors would thank Universitas Sumatera Utara (USU) for the facilities to perform this work including the characterization.

\section{Notes and references}

1 E. M. Ahmed, J. Adv. Res., 2015, 6, 105-121.

2 C. Nam, T. J. Zimudzi, G. M. Geise and M. A. Hickner, ACS Appl. Mater. Interfaces, 2016, 8, 14263-14270.

3 L. Saunders and P. X. Ma, Macromol. Biosci., 2019, 19, e1800313.

4 V. Pertici, C. Pin-Barre, C. Rivera, C. Pellegrino, J. Laurin, D. Gigmes and T. Trimaille, Biomacromolecules, 2019, 20, 149-163.

5 H. Chen, J. Cheng, L. Ran, K. Yu, B. Lu, G. Lan, F. Dai and F. Lu, Carbohydr. Polym., 2018, 201, 522-531.

6 Z. Wei, J. He, T. Liang, H. Oh, J. Athas, Z. Tong, C. Wang and Z. Nie, Polym. Chem., 2013, 4, 4601.

7 X. Li, Q. Yang, Y. Zhao, S. Long and J. Zheng, Soft Matter, 2017, 13, 911-920.

8 C. Shao, M. Wang, H. Chang, F. Xu and J. Yang, ACS Sustainable Chem. Eng., 2017, 5, 6167-6174.

9 Y. Yakabe, T. Sano, H. Ushio and T. Yasunaga, Chem. Lett., 1980, 9, 373-376.

10 W. Sim, R. T. Barnard, M. A. T. Blaskovich and Z. M. Ziora, Antibiotics, 2018, 7, 93.

11 S.-H. Min, J.-H. Yang, J. Y. Kim and Y.-U. Kwon, Microporous Mesoporous Mater., 2010, 128, 19-25.

12 N. T.-P. Nguyen, L. V.-H. Nguyen, N. T. Thanh, V. V. Toi, T. Ngoc Quyen, P. A. Tran, H.-M. David Wang and T.-H. Nguyen, Mater. Lett., 2019, 248, 241-245.

13 M. Montazer, A. Shamei and F. Alimohammadi, Prog. Org. Coat., 2012, 75, 379-385.

14 A. D. Jenkins, P. Kratochvil, R. F. T. Stepto and U. W. Suter, Pure Appl. Chem., 1996, 68, 2287-2311.

15 A. P. Rokhade, S. A. Agnihotri, S. A. Patil, N. N. Mallikarjuna, P. V. Kulkarni and T. M. Aminabhavi, Carbohydr. Polym., 2006, 65, 243-252.

16 M. K. Satapathy, W. H. Chiang, E. Y. Chuang, C. H. Chen, J. L. Liao and H. N. Huang, PeerJ, 2017, 5, e3498.

17 E. O. Akala, P. Kopeckova and J. Kopecek, Biomaterials, 1998, 19, 1037-1047.

18 J. E. Elliott, M. Macdonald, J. Nie and C. N. Bowman, Polymer, 2004, 45, 1503-1510. 
19 E.-E. Hago and X. Li, Adv. Mater. Sci. Eng., 2013, 2013, 1-8. 20 Q. Xing, K. Yates, C. Vogt, Z. Qian, M. C. Frost and F. Zhao, Sci. Rep., 2014, 4, 4706.

21 G. Russell, J. Photogr. Sci., 2016, 15, 151-157.

22 R. Goering, H. Dockrell, M. Zuckerman and P. Chiodini, Mims' Medical Microbiology and Immunology, Elsevier, 6th edn, 2018.

23 J. L. Clement and P. S. Jarrett, Met.-Based Drugs, 1994, 1, 467482.
24 Y. N. Slavin, J. Asnis, U. O. Hafeli and H. Bach, J. Nanobiotechnol., 2017, 15, 65.

25 M. L. Guerinot, Annu. Rev. Microbiol., 1994, 48, 743-772.

26 C. Vilcheze, T. Hartman, B. Weinrick and W. R. Jacobs Jr, Nat. Commun., 2013, 4, 1881.

27 D. Wang, F. Peng, J. Li, Y. Qiao, Q. Li and X. Liu, Mater. Today, 2017, 20, 238-257. 